\title{
Herbig Ae/Be Visual Binaries
}

\author{
Jérôme Bouvier \& Patrice Corporon \\ Observatoire de Grenoble, Université J. Fourier, B.P. 53, 38041 \\ Grenoble Cedex 9, France
}

\begin{abstract}
We present the results of a high-angular resolution spectroimaging survey of a sample of isolated Herbig Ae-Be (HAeBe) stars aimed at searching for close companions. The fraction of wide binaries is found to be significantly higher in HAeBe stars than in solar-mass field dwarfs, and suggests a companion star fraction possibly larger than 1 . The spectral energy distribution of the primaries and secondaries is derived independently from multi-wavelength resolved photometry of the systems. It is found that young low-mass companions usually do not exhibit infrared excesses, which suggests that the lifetime of their circumstellar disk might have been shortened by the influence of the massive primary.
\end{abstract}

\section{Introduction}

In the past decade, observational investigations of multiple systems among young low-mass stars have significantly modified our perception of the star formation process. In order to settle the most pressing issues, most studies so far have been focusing onto solar-type and lower-mass stellar populations. As the physical implications of the large number of multiple systems found in these populations are being drawn, the study of more massive binaries will provide new constraints on models of binary formation and evolution. How the binary frequency and the distributions of orbital period and mass ratio varies as a function of the primary mass will help to choose between current models.

In this contribution, we report the results of a high-angular resolution imaging survey of a sample of young massive stars aimed at improving the binary statistics among Herbig $\mathrm{Ae}-\mathrm{Be}(\mathrm{HAeBe})$ stars and at investigating the spectrophotometric properties of the components of massive multiple systems. Implications for binary formation and the evolution of the circumstellar environment in these systems are briefly discussed.

\section{The Sample}

The magnitude-limited $(\mathrm{R} \leq 14)$ sample consists of $63 \mathrm{HAeBe}$ candidates selected from Thé, DeWinter \& Pérez's (1994) catalogue. Observations at visual and near-IR wavelengths were obtained with CFHT's adaptive optics system PUEO in the Northern hemisphere and with ESO's adaptive optics system ADONIS in the Southern hemisphere. Both systems deliver diffraction-limited images at $\mathrm{K}$ with a FWHM of $\sim 0.1^{\prime \prime}$. 

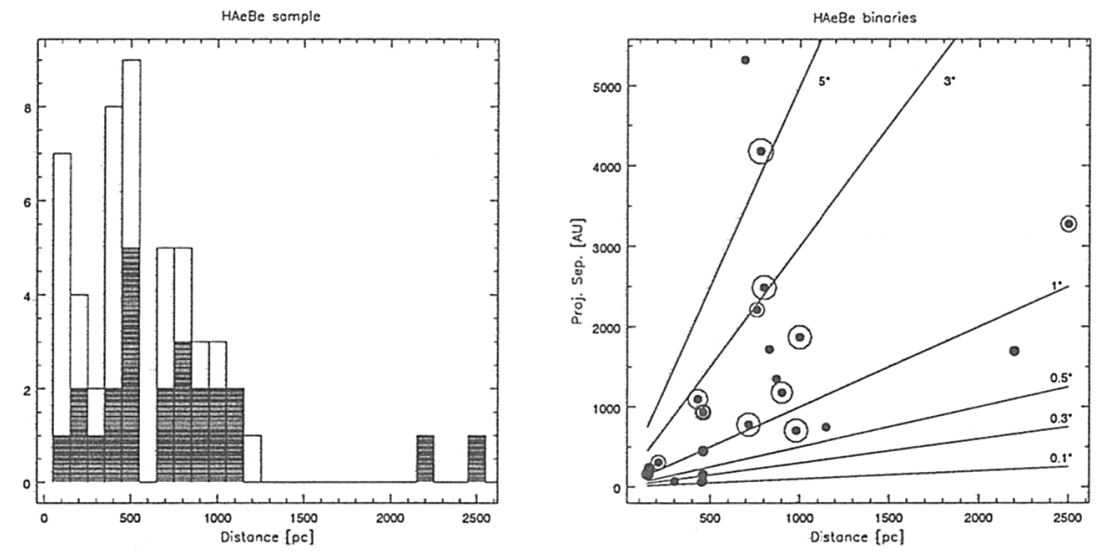

Figure 1. Left: Distance distribution of the observed intermediatemass pre-main sequence stars (bar histogram). The dashed histogram indicates the number of binary systems detected in each distance bin. Right: Projected separation of binary components as a function of distance. Each binary is represented by a filled dot and the size of the surrounding circle is indicative of the flux ratio between the primary and the secondary. The lines indicate the locus of constant angular resolution in this diagram.

The sample is inhomogeneous in several ways, which complicates the derivation of unbiased binary statistics for $\mathrm{HAeBe}$ stars. While most of the stars listed in Thé et al. (1994) are probably bona fide intermediate-mass, pre-main sequence stars, the authors warn about a possible contamination of the catalogue by non$\mathrm{HAeBe}$ stars (e.g. $\mathrm{B}[\mathrm{e}]$ main sequence objects). All stars in our sample were selected from the best $\mathrm{HAeBe}$ candidates of this catalogue. Another difficulty lies in the wide range of distances covered by the stars in the sample, from less than $100 \mathrm{pc}$ up to $\sim 2 \mathrm{kpc}$. In addition, the distance of some candidates is unknown. Since the survey is angular resolution limited, the range of semi-major axes it probes varies with distance. Finally, $\mathrm{HAeBe}$ stars occur in a variety of environments, from being isolated to belonging to small clusters (Testi et al. 1998), and the impact of the environment on massive binary formation and evolution is unknown (see Patience \& Duchêne, this volume). Thus, deriving reliable binary statistics for HAeBe stars is not as straightforward as for T Tauri stars, not mentioning the large contrast between a low-mass companion and a massive primary which involves significant completeness corrections to be applied to the observed fraction of binaries.

\section{Binary Statistics}

Among the $63 \mathrm{HAeBe}$ stars observed, 27 are found to have a companion at a separation less than $3^{\prime \prime}$. Figure 1a shows the distribution of distance for 50 stars of the sample (13 other stars have unknown distances). In each distance bin, the dashed histogram illustrates the fraction of detected binaries. Since 
most sample stars are located at less than $1.2 \mathrm{kpc}$, we will exclude the 2 most remote systems from the analysis below. Figure $1 \mathrm{~b}$ illustrates the distribution of detected binaries in a separation-distance diagram. The field-of-view of most of our high angular resolution images is $6^{\prime \prime}$. Therefore, we will restrict the computation of binary frequency to systems in the separation range $\sim 0.1-3.0^{\prime \prime}$. By doing so, we consider as physical binaries only systems with a projected semi-major axis less than $2000 \mathrm{AU}$.

Within these limits, the sample contains 44 HAeBe stars with known distances and a spectral type between B0 and A9 of which 18 have a companion, i.e., a raw binary fraction (BF) of $41 \pm 10 \%^{1}$. Adding to this subsample 10 B0-A9 primaries and 3 systems with unknown distance yields a BF of $39 \pm 9 \%$. Within Poisson uncertainties, the $\mathrm{BF}$ is found to be the same among $\mathrm{B} 0-\mathrm{B} 7$ primaries $\left(7-20 \mathrm{M}_{\odot}, \mathrm{BF}=36 \%\right)$ and among $\mathrm{B} 9-\mathrm{A} 9$ ones $\left(2-3 \mathrm{M}_{\odot}, \mathrm{BF}=42 \%\right)$. These results are consistent with those derived previously from a smaller sample (that partly overlaps with ours) by Leinert, Richichi \& Haas (1997).

The orbital periods have been estimated from the total mass of the system and statistically correcting for the projection of the semi-major axis. Figure 2 shows the orbital period distribution of the detected binaries with known distance. The distribution of orbital periods for G-dwarf binaries (Duquennoy \& Mayor 1991, DM91) is also shown for comparison. Note that the DM91 distribution is complete in each $\log P$ bin down to a mass ratio of 0.1 while the distribution for $\mathrm{HAeBe}$ stars has not been corrected for incompleteness. Since we have most likely missed a number of companions at these separations (see below), the HAeBe histogram is a lower limit to the true binary frequency in each bin. Therefore, between $\log P=5.0-7.0$ days, the binary excess among $\mathrm{HAeBe}$ stars relative to $\mathrm{G}$ dwarfs appears to be at least a factor of two.

We attempted to compute the level of incompleteness on the statistics above, i.e., to estimate the number of companions that we have missed due to their proximity to the primary and/or their low luminosity. We have assumed that the orbital period distribution of HAeBe binaries has the same Gaussian shape as that of G dwarf binaries (DM 91, which is in fact nearly flat in the restricted $\log P$ range investigated here), and that the mass-ratio distribution is either the same as for $\mathrm{G}$ dwarfs (case A) or derives from random pairing of lowmass companions to massive primaries according to an IMF prescription (case B). For the latter case, we adopted a power-law IMF with a Salpeter exponent of 2.3 for stars more massive than $1.0 M_{\odot}$ and of 1.0 for lower-mass stars. With these assumptions, and taking into account the (separation-dependent) detection limit of adaptive optics images, we estimate that we detected about $60-80 \%$ (case A or B) of the companions down to a mass ratio of 0.1. However, down to a companion mass of $0.1 \mathrm{M}_{\odot}$ (which corresponds to a mass ratio of 0.01 for a $10 \mathrm{M}_{\odot} \mathrm{B}$-type primary), only about $25 \%$ of the companions to $\mathrm{B} 0-\mathrm{B} 7$ primaries are detected (case B).

Since the raw binary frequency is of order of $40 \%$ in the range $\log P=$ $5.0-7.0 d$, correcting for incompleteness would yield a true BF of about $50-60 \%$ in this orbital period range. For comparison, the BF of $\mathrm{G}$ dwarfs over the same orbital period range is only $20 \%$. Thus, if the distribution of orbital period is at

\footnotetext{
${ }^{1}$ Not very different from the BF deduced for the whole sample, $27 / 63=43 \pm 8 \%$
} 

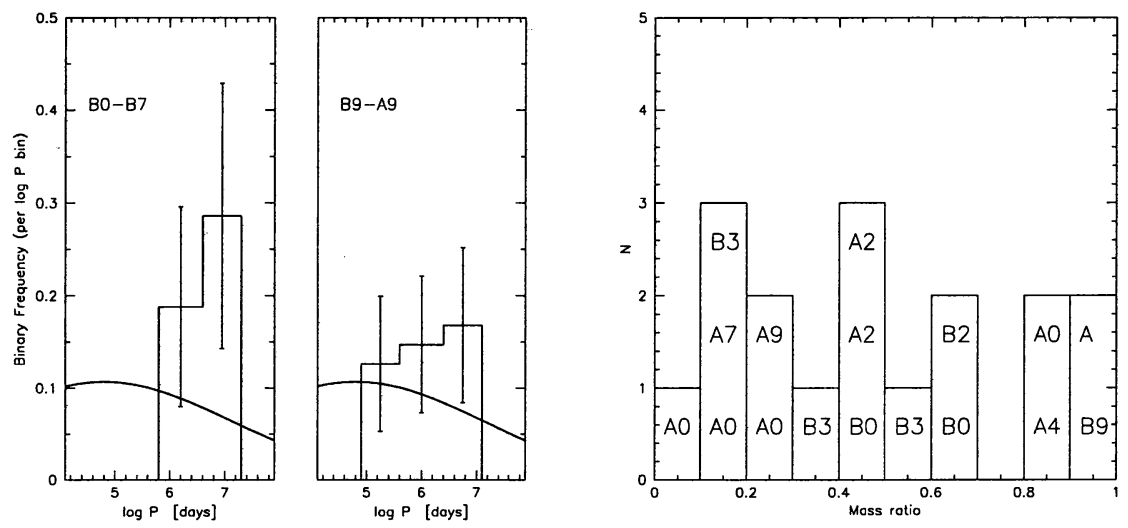

Figure 2. Left: the distribution of orbital periods for binaries detected in our survey (histogram). Vertical bars illustrate Poisson noise. The distribution of orbital periods for G dwarf binaries (from DM91) is shown for comparison (solid curve). Right: the distribution of mass ratios for binaries with B-type and A-type primaries. The spectral type of the primary is indicated. Mass ratios were derived from flux ratios with an uncertainty of about $20 \%$.

least approximately the same for HAeBe binaries and G dwarfs, extrapolating this result over the whole range of semi-major axes would suggest that $\mathrm{HAeBe}$ stars have on average more than one companion. A similarly high companion star fraction is found among the massive stars of the Orion Trapezium cluster (see Preibisch et al. 1999, and this volume) as well as in OB stars belonging to the NGC 6611 cluster (Duchêne 2000).

While the most complete binary statistics on the main sequence holds for G dwarfs, it would be more meaningful to compare the results obtained on $\mathrm{HAeBe}$ binaries to the binary fraction among massive dwarfs. Unfortunately, the binary statistics in massive main sequence stars is currently rather incomplete, being derived from a variety of techniques that suffer strong biases. Thus, the $\mathrm{BF}$ among massive dwarfs has been reported as being similar to that of lowmass dwarfs by some authors (e.g. Abt 1983, McAlister et al. 1993) and as much as 3 times larger by others (Abt, Gomez \& Levy 1990, Mason et al. 1998). Considering these uncertainties, a comparison of the PMS and MS binary frequency among massive stars is probably premature.

Figure 2 also shows the mass ratio distribution deduced for part of the binary sample. For the systems with B-type primaries, mass-ratios were derived from the flux ratio in the V-band using the MS mass-luminosity relationship. For systems with A-type primaries, the mass ratio was obtained from the flux ratio in the J-band (to avoid the complication of near-IR excesses in the K-band and possible veiling at visual wavelengths in T Tauri-type companions) using the mass-luminosity relationship provided by Siess et al. (2000) PMS models at an age of 5 Myr. Mass ratio could be derived only for part of the binary sample since several had no measurements in the $\mathrm{V}$ or $\mathrm{J}$ bands. The distribution is not 
significantly peaked, neither at low nor at high q values, and is in fact consistent with being flat regardless of the mass of the primary.

\section{Spectrophotometric Properties of the Binary Systems}

Combining differential photometry between the primary and the secondary derived from the adaptative optics images with absolute photometry for the whole system listed in the literature, the spectral energy distribution (SED) of each component of the HAeBe binaries has been derived from optical to near-IR wavelengths. Assuming the same visual extinction for both components, dwarf-like photospheres can then be fitted to the optical part of the companion's SED in order to estimate its spectral type. Examples of these SED and spectral type derivation are shown in Figures 3 and 4.

We can classify the HAeBe binaries in 3 broad groups. The first group consists of binaries with 2 massive Herbig Be stars (e.g. MWC 166, GU CMa, Hen 3-225), the second group of a Herbig Be primary associated with a lowmass T Tauri-type companion with a spectral type G or K (e.g. V380 Ori, MWC 147, MWC 361), and the third group consists of a Herbig Ae primary with a T Tauri type companion (Figure 4). Although we did not find any very low-mass companion, with a spectral type $M$, this is probably merely due to our detection limits.

Binaries with a B-type primary are illustrated in Figure 3. It is seen that none of the companions, Be and TT stars alike, exhibit a significant near-IR excess compared to a dwarf photosphere, except perhaps V380 Ori B at K. In addition, when the companion is a massive $\mathrm{B}$ star, the B-type primary also lacks a significant near-IR excess. In contrast, massive primaries with TT companions systematically exhibit excess flux in the near-IR. A similar trend is observed for binaries with A-type primaries (Figure 4): while the A-type primaries usually exhibit strong near-IR excesses, the TT companions seem to systematically lack the signature of circumstellar dust.

The spectrophotometric properties of $\mathrm{HAeBe}$ binaries thus suggest that the circumstellar environment of the low-mass companions may be strongly affected by the presence of a massive primary. A number of physical mechanisms might affect the formation and evolution of circumstellar material around a young low-mass star located in the vicinity of, and gravitationally bound to, a more massive and luminous star. This includes, at least, competitive accretion, tidal truncation, and photoevaporation of the disk.

Competitive accretion: assuming that HAeBe binaries result from the fragmentation of a molecular cloud core, thus leading to the formation of two protostellar cores with unequal mass, the accretion of residual gas onto the protostellar cores may be preferentially directed towards the most massive one whose potential well is deeper. This would result in a massive circumprimary disk and a relatively low-mass, and therefore shorter-lived circumsecondary one. This process will probably be more important for relatively wide binaries formed in small-N clusters (Bonnell et al. 1997). Although it could qualitatively account for the systematic lack of near-IR excesses in the low-mass companions of Herbig Ae-Be stars, this process would however not necessarily explain the lack of 

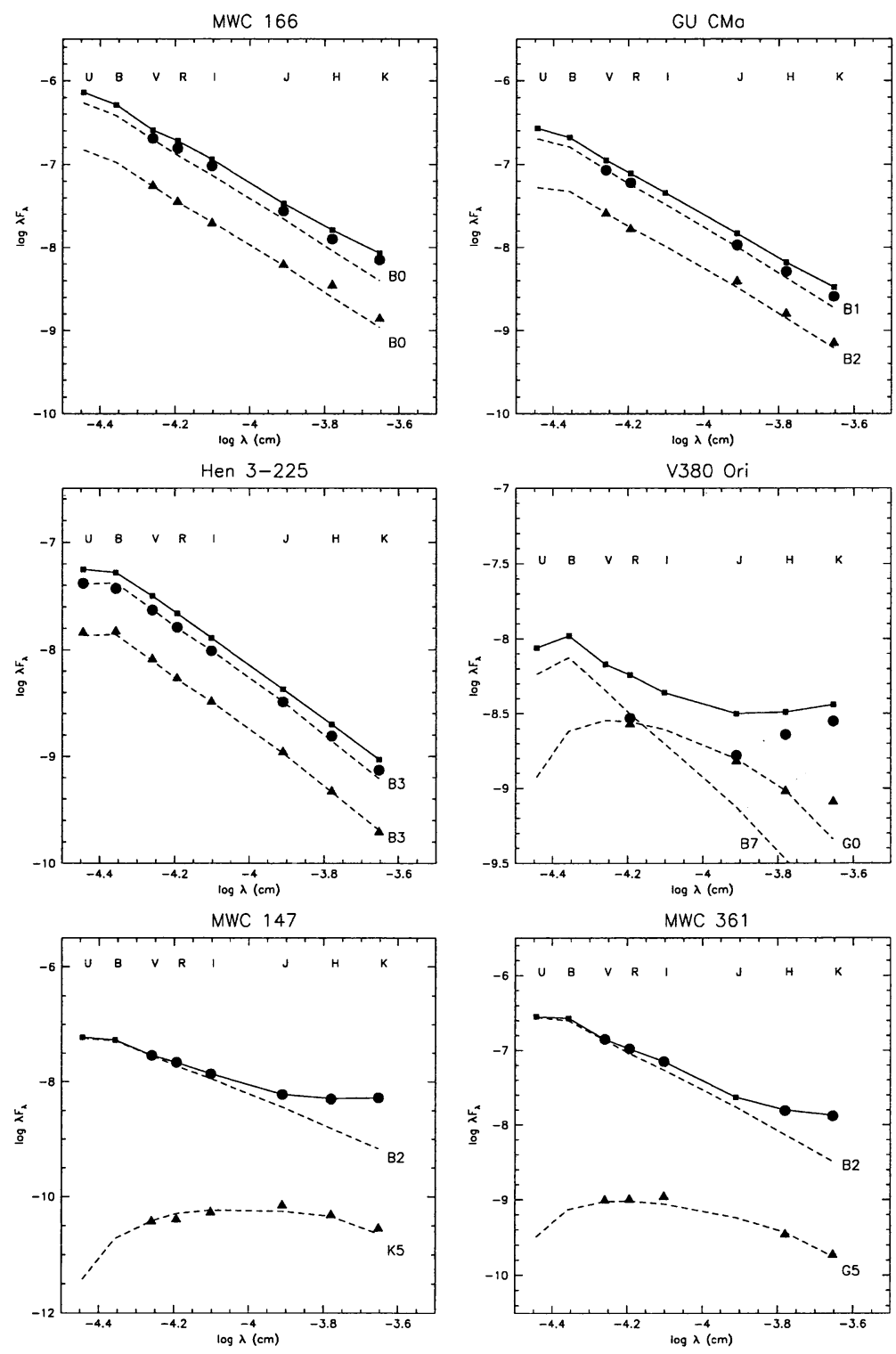

Figure 3. The spectral energy distribution of pre-main sequence binaries with a B-type primary. The SED of the primary is shown as filled dots and of the secondary as filled triangles. Filled squares represent the total flux measured for the whole system. A reddenned photosphere (dashed line) has been fitted to each of the components at visual wavelengths. The spectral type of the primary is either taken from the literature or derived from the SED fit. The secondary's spectral type is derived from the SED fit and indicated. The same $A_{V}$ is assumed for the primary and the secondary and was taken from the literature. 

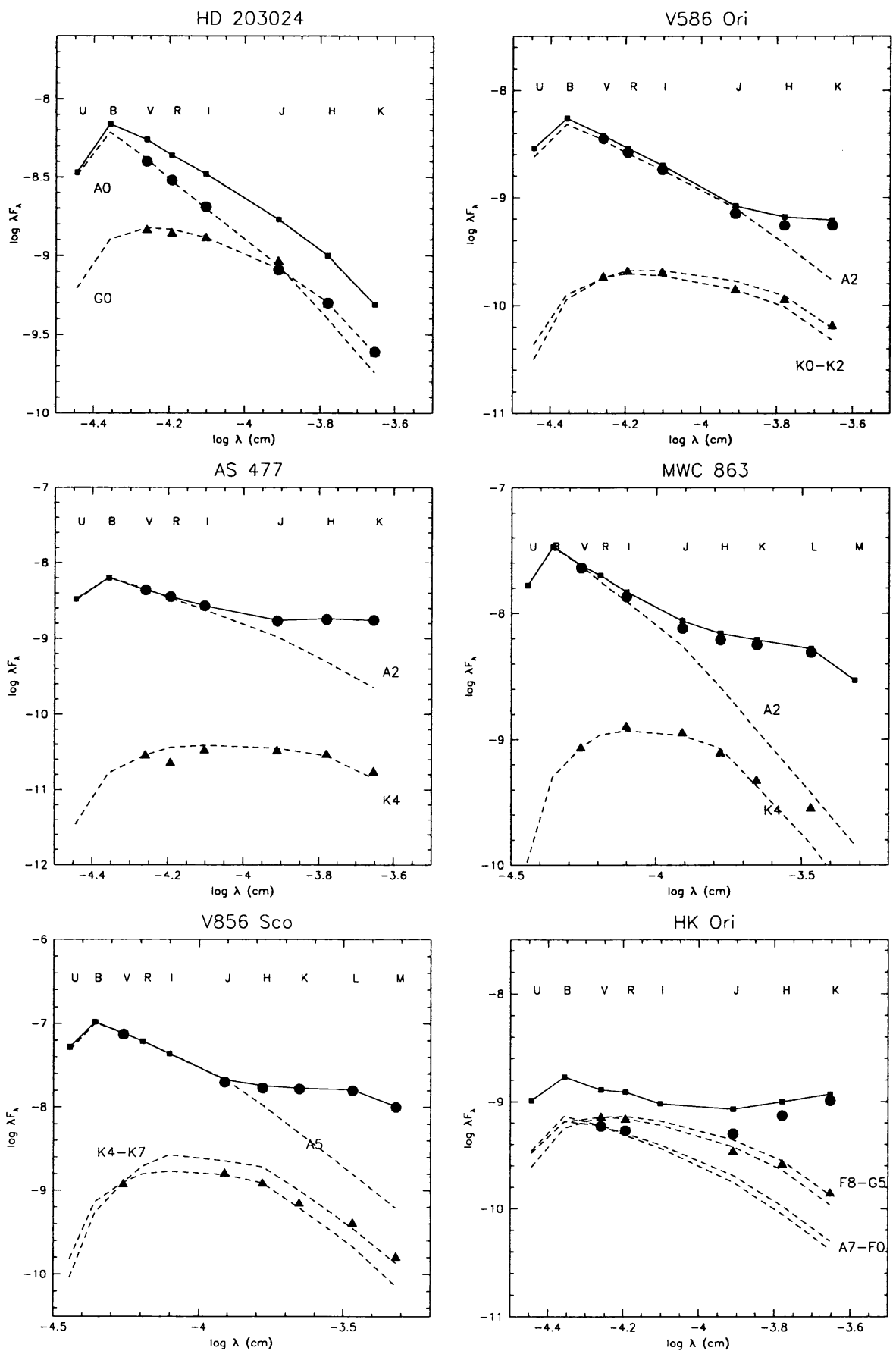

Figure 4. Same as Figure 3 but for binaries with A-type primaries. 
near-IR excesses in massive binaries with a mass ratio close to unity (e.g. the Be-Be systems).

Tidal truncation and circumbinary accretion: for the tightest systems, with a separation of order of the radius of the residual infalling envelope, circumstellar (CS) disks are tidally truncated and a circumbinary (CB) disk/envelope may result. The relative lifetime of the CS disks then depends upon their size and on their differential replenishment as they accrete CB material. The latter process heavily depends upon the properties of the system and its environment (initial mass ratio, eccentricity, specific angular momentum of the CB material, cf. Bate 2000). Qualitatively, however, tidal truncation in unequal mass systems should result in a smaller disk around the secondary which may thus evolve on a shorter timescale $\left(t_{\nu} \propto R^{2} / \nu\right)$. Even in nearly equal-mass binaries (e.g. the Be-Be systems) truncation effects would reduce the disks radius and thus their lifetime, possibly accounting for the lack of NIR excesses in both components. In this respect, MWC 1080, an unusual B2-B2 binary in which both components do exhibit strong infrared excesses, might be a very young system which has not yet depleted its circumstellar disks. The detection of an associated molecular outflow supports an extremely young age for this system (Yoshida et al. 1991).

Photoevaporation: compared to T Tauri stars, Herbig Be-Ae stars emit a much larger fraction of their luminosity in the far-UV (FUV) range. FUV photons impacting onto a circumstellar disk heat its surface to a temperature of about $10^{3} \mathrm{~K}$ which leads to the development of a massive disk wind. According to models (e.g. Johnstone et al. 1998), FUV irradiated disks thus photoevaporate on a relatively short timescale $\left(\sim 10^{5} \mathrm{yrs}\right)$ as soon as the incident FUV photon flux is of order of $10^{4} G_{o}$ or larger, where $G_{o}$ is the average UV photon flux in the local insterstellar medium. Such a radiation field is produced for instance by the hot (O-type) Orion Trapezium stars up to a distance of about 1pc, which is the typical size of the region over which proplyds are observed (Störzer \& Hollenbach 1999 and references therein). For slightly colder stars, such as Herbig Be-Ae stars, the UV photon flux is greatly reduced. Nevertheless, the distance up to which these stars produce the minimum FUV flux required to photoevaporate disks is still of order of $10^{4}$ AU from a B3 star and about 500 $\mathrm{AU}$ from an A0 star. Hence, in at least some of the systems detected during our survey, especially those with massive primaries, the (projected) separation and the primary's luminosity are such that photoevaporation of the circumsecondary disk might have already occurred as a result of it being irradiated by the primary's FUV photon flux. This could account for the lack of near-IR excesses in at least some of the companions of these systems. In most cases, however, the primaries still exhibit near-IR excesses indicating that they have not photoevaporated their own disks. Although this result casts some doubt on this interpretation, there might be a couple of reason why circumprimary disks might survive photoevaporation longer than circumsecondary ones. Firstly, only the outer parts of the disks are photoevaporated by this process, beyond the "gravitational" radius defined as $r_{g} \sim(0.2-0.5) \cdot G M_{\star} / a^{2}$, where $a$ is the sound speed in the gas (typically a few $\mathrm{km} / \mathrm{s}$ ). This radius is of order of $30 \mathrm{AU}$ for a $1 M_{\odot}$ secondary and about $300 \mathrm{AU}$ for a $10 M_{\odot}$ primary. Secondly, dust opacity in the disk atmosphere and in the disk wind may significantly lower the incident UV flux that reaches the outer part of the circumprimary disk (Hollenbach et al. 
1994) while, unless the circumprimary and circumsecondary disks are coplanar, FUV photons may more easily escape in the direction of the companion.

The spectrophotometric properties of resolved components of HAeBe binaries thus hold clues to the formation and evolution of these systems. Several processes may be at work, either simultaneously or not, depending on the binary properties, and the analysis is further complicated by the fact that some of the primaries and secondaries may themselves be multiple systems, such as spectroscopic binaries (cf. Corporon \& Lagrange 1999). Interestingly enough, the SEDs of $\mathrm{HAeBe}$ binaries reveal a trend which is opposite to that observed for $\mathrm{T}$ Tauri binaries. Several studies have shown that the components of T Tauri binaries have "correlated" near-IR SEDs, with either both or none of the components exhibiting an infrared excess. Mixed pairs, with one component exhibiting a near-IR excess and the other not, are very rare among TT binaries (e.g. Prato \& Simon 1997, Ghez et al. 1997, Duchêne et al. 1999). In contrast, Herbig AeBe binaries predominantly consist of such mixed pairs. Since HAeBe and TT binaries seem to have otherwise similar properties (projected separations, mass ratio distribution), it is tempting to ascribe this difference to the impact of the luminous primary onto the secondary's environment (e.g. photoevaporation) rather than to dynamical effects in binary systems (e.g. disk truncation, differential circumbinary accretion, etc...).

\section{Conclusions}

Multiple systems appear to be extremely common among intermediate-mass premain sequence stars. Moreover, the binary fraction appears to be equally high among isolated $\mathrm{HAeBe}$ and among massive stars in dense clusters. In contrast, at lower masses, fewer $\mathrm{T}$ Tauri binaries are found in dense clusters than in associations (see Patience \& Duchêne, this volume). This difference may have some implications for the binary formation process. According to current (and still somewhat speculative) ideas, the fragmentation of molecular cloud cores may initially lead to a protobinary fraction close to $100 \%$. In dense clusters, a number of the protobinaries are disrupted by gravitational encounters (e.g. Kroupa, Petr, \& McCaughrean 1999), which results in a lower binary fraction in dense environments (such as Orion) than in loose associations (such as Taurus) at an age of about $1 \mathrm{Myr}$. That massive binaries do not seem to show the same density-related binary frequency may simply reflect the increased hardness of these massive systems compared to TT systems, which make them more difficult to disrupt. If correct, one would expect a different distribution of orbital periods between $\mathrm{HAeBe}$ and TT binaries, with a larger fraction of wide systems in the former than in the latter, a prediction that is not inconsistent with the data at hand.

Alternatively, models of dynamical capture in small-N protoclusters predict an increase of the binary frequency with the mass of the primary. Current results tend to agree with this prediction since in most star forming regions the binary fraction among low-mass stars is statistically consistent with that of G dwarfs, i.e., of order of $60 \%$ (with, however, the noticeable exception of the Taurus cloud), while it appears to be a factor of at least 2 higher among Herbig stars. Models of dissipative interactions in small-N protoclusters also predict a 
companion mass distribution that is invariant with primary mass (McDonald \& Clarke 1995). Unfortunately, due to the limited detectability levels and reduced size of the samples, current studies do not quite allow a statistically reliable determination of the distribution of mass ratios in intermediate-mass binary systems.

Finally, the photospheric-like spectral energy distribution of the low-mass companions of Herbig stars suggests that the luminous primary might significantly affect the evolution of the circumsecondary and possibly circumbinary environment. Additional modelling and observations, especially resolved spectroscopy of the systems, will be needed to fully understand the formation and fate of the circumstellar environment in young massive multiple systems.

\section{References}

Abt, H. A. 1983, ARA\&A, 21, 343

Abt, H. A., Gomez A. E., \& Levy, S. G. 1990, ApJS, 74, 551

Bate, M. R. 2000, MNRAS, 314, 33

Bonnell, I., Bate, M. R., Clarke, C. J., \& Pringle, J. E. 1997, MNRAS, 285, 201

Corporon, P., \& Lagrange, A.-M. 1999, A\&AS, 136, 429

Duchêne, G. 2000, Ph.D. Thesis, Université Grenoble

Duchêne, G., Monin, J.-L., Bouvier, J., \& Ménard, F. 1999, A\&A, 351, 954

Duquennoy, A., \& Mayor, M. 1991, A\&A, 248, 485

Ghez, A. M., White, R. J., Simon, M. 1997, ApJ, 490, 353

Hollenbach, D., Johnstone, D., Lizano, S., \& Shu, F. 1994, ApJ, 428, 654

Johnstone, D., Hollenbach, D., \& Bally, J. 1998, ApJ, 499, 758

Kroupa, P., Petr, M. G., \& McCaughrean, M. J. 1999, New Astron., 4, 495

Leinert, Ch., Richichi, A., \& Haas, M. 1997, A\&A, 318, 472

McAlister, H. A., Mason, B. D., Hartkopf, W. I., Shara, M. M. 1993, AJ, 106, 1639

McDonald, J. M., Clarke, C. J. 1995, MNRAS, 275, 671

Mason, B. D., Gies, D. R., Hartkopf, W. I., et al. 1998, AJ, 115, 821

Prato, L., \& Simon, M. 1997, ApJ, 474, 455

Preibisch, Th., Balega, Y., Hofmann, K. H., et al. 1999, New Astron. 4, 531

Siess, L., Dufour, E., \& Forestini, M. 2000, A\&A, in press

Störzer, H., \& Hollenbach, D. 1999, ApJ, 515, 669

Testi, L., Palla, F., \& Natta, A. 1998, A\&AS, 133, 81

Thé, P. S., de Winter, D., \& Pérez, M. R. 1994, A\&AS, 104, 315

Yoshida, S., Kogure, T., Nakano, M., et al. 1991, PASJ, 43, 363 